\title{
Erratum: Glucose-responsive insulin by molecular and physical design
}

Naveed A. Bakh, Abel B. Cortinas, Michael A. Weiss, Robert S. Langer, Daniel G. Anderson, Zhen Gu, Sanjoy Dutta and Michael S. Strano

Nature Chemistry 9, 937-943 (2017); published online 22 September 2017; corrected after print 24 November 2017.

In the version of this Perspective originally published, the affiliations for authors Zhen Gu and Sanjoy Dutta were not correct, they should have read:

Zhen $\mathrm{Gu}^{3,4,5}$, Sanjoy Dutta ${ }^{6}$

${ }^{3}$ Joint Department of Biomedical Engineering, University of North Carolina at Chapel Hill and North Carolina State University, Raleigh, North Carolina 27695, USA. ${ }^{4}$ Pharmacoengineering and Molecular Pharmaceutics Division, Eshelman School of Pharmacy, University of North Carolina at Chapel Hill, Chapel Hill, North Carolina 27599, USA. ${ }^{5}$ Department of Medicine, University of North Carolina School of Medicine, Chapel Hill, North Carolina 27599, USA. ${ }^{6}$ JDRF International, New York, New York 10004, USA. 